\title{
A free boundary problem arising in a model for shallow water entry at small deadrise angles
}

\author{
S. D. HOWISON ${ }^{1}$ and J. M. OLIVER ${ }^{2}$ \\ ${ }^{1}$ Mathmatical Institute, 24 - 29 St. Giles', Oxford OX1 3LB, UK \\ ${ }^{2}$ School of Mathematical Sciences, University of Nottingham, Nottingham NG7 2RD, UK
}

(Received 23 June 2004)

\begin{abstract}
A free boundary problem arising in a model for inviscid, incompressible shallow water entry at small deadrise angles is derived and analysed. The relationship between this novel free boundary problem and the well-known viscous squeeze film problem is described. An inverse method is used to construct explicit solutions for certain body profiles and to find criteria under which the splash sheet can 'split'. A variational inequality formulation, conservation of certain generalized moments and the Schwarz function formulation are introduced.
\end{abstract}

\section{Introduction}

The impact of a rigid body on liquid is an important free boundary problem with applications ranging in scale from asteroid impact to droplet motion. The severe nonlinearities involved mean that even the basic problem in which the liquid is inviscid and incompressible, the flow starts from rest and is therefore irrotational, and the effects of gravity, surface tension and air cushioning are neglected, is only amenable to exact theoretical treatment in special cases such as that of wedge impact [4]. In this paper, we consider an approximate model arising from Wagner theory [17] for normal impact of a blunt body under the assumptions stated above. Our model describes the normal impact of a blunt body on a shallow layer of liquid of uniform initial thickness. We are concerned with the situation in which the curvature of the body is sufficiently small (i.e. at small deadrise angles) that, even when the body penetration below the undisturbed waterline is small, the lateral extent of the part of the body below the undisturbed waterline is large compared to the water depth. Such a situation arises whenever the body shape and locations, in dimensionless cartesian coordinates $\left(x^{*}, y^{*}, z^{*}\right)$ with $z^{*}$ normal to the water layer $-1<z^{*}<0$ and with a suitable scaling of time $t$, are given by

$$
z^{*}=\epsilon\left(f\left(\delta x^{*}, \delta y^{*}\right)-s(t)\right)
$$

where $\epsilon \dot{s}(t)$ is the impact velocity and $\epsilon \ll 1, \delta \ll 1$ are independent small parameters.

In such cases, as shown in [7], the impact takes place over four stages. In the initial stage the body penetration is sufficiently small that the Wagner model [5] for impact on a fluid of infinite depth provides a good description. The salient features of this model, as 


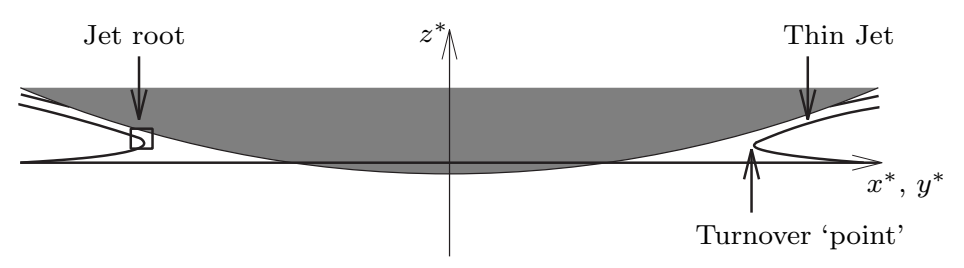

Figure 1. Schematic of infinite depth entry at small at small deadrise angles

sketched in figure 1, are that the free surface 'turns over' in the very small regions which are very close to the body, and these turnover regions are the roots of a thin high-velocity splash sheet which is ejected from under the body. This feature allows a linearized model to be formulated as a "codimension-two" free boundary problem $[\mathbf{6}]$ in which the velocity potential satisfies Laplace's equation with boundary conditions which switch across the points (in two-dimensions) or curve (in three-dimensions) corresponding to the small turnover regions; the region under the body and inside the turnover curve is termed the 'contact set'. Lastly the condition that the turnover regions are close to the body leads to a 'law of motion' for the evolution of the turnover curve and a prediction of the velocity and thickness of the splash sheet at its root. In the second stage of the evolution, the contact set is comparable in lateral extent to the water depth, so the effect of the base is felt at leading order. The third stage, in which the lateral extent of the contact set is much greater than the water depth, is the subject of this paper, while the fourth stage, in which the penetration is no longer much smaller than the depth, can be described by 'Korobkin theory' [7, 9].

We now consider in more detail the third stage, in which for a body (1.1), the penetration is $\mathrm{O}(\epsilon)$ while the lateral extent of the contact set is $\mathrm{O}(1 / \delta)$ relative to the water depth. We then have a large aspect ratio approximation of the codimension-two free boundary problem for stage two of the impact, as described in detail in [6] for other such problems. In this case, the flow beneath the body is an 'inviscid squeeze film', and scaling $\left(x^{*}, y^{*}\right)=\delta^{-1}(x, y)$ the velocity is approximately $(u, v, 0)$ where $u=\phi_{x}, v=\phi_{y}$ for a suitably scaled velocity potential $\phi(x, y, t)$, which, by mass conservation, satisfies

$$
\nabla^{2} \phi=\dot{s}
$$

in the contact set, where $\nabla$ is the two-dimensional gradient operator. For later convenience we denote the turnover curve $\Gamma(t)$, which we assume is expanding, by

$$
t=\omega(x, y)
$$

so the contact set $\Omega(t)$ is $\omega(x, y)<t$ and the initial condition is simply $\omega(0,0)=0$ on taking the initial contact to occur at the origin. As shown in $[\mathbf{7}, \mathbf{1 1}]$, the flow velocity outside the contact set and the neighourhood of the small turnover region is exponentially small, and the analysis of the flow near to the turnover curve shows that the appropriate conditions there are

$$
\begin{aligned}
\phi & =0 \\
\phi_{n} & =v_{n}(f(x, y)-s(t)),
\end{aligned}
$$

where $\phi_{n}$ denotes the outward normal derivative of $\phi$ on the turnover curve, which 
has outward normal velocity $v_{n}$. Equations (1.2) - (1.4) for prescribed $f(x, y)$ and $s(t)$ constitute the free boundary value problem that is the subject of this paper. The onedimensional specialisation of this problem (corresponding to two-dimensional flow under the impactor), in which the contact set has the form $d_{-}(t)<x<d_{+}(t)$ and the body profile is $f(x)-s(t)$ where $f(0)=0$ and $f^{\prime}(x) \lessgtr 0$ for $x \lessgtr 0$, can be solved explicitly; integrating

$$
\phi_{x x}=\dot{s} \quad \text { on } \quad d_{-}(t)<x<d_{+}(t)
$$

subject to

$$
\phi=0, \quad \phi_{x}=\dot{d}_{ \pm}(f(x)-s(t)) \quad \text { at } \quad x=d_{ \pm}(t),
$$

we find

$$
\phi=\frac{\dot{s}}{2}\left(x-d_{-}\right)\left(x-d_{+}\right) \quad \text { where } \quad \int_{d_{-}}^{d_{+}}(f(\xi)-s(t)) \xi^{j} \mathrm{~d} \xi=0 \text { for } j=0,1,
$$

the latter integrals respresenting overall mass and $x$-momentum conservation. We therefore have two equations for $d_{ \pm}(t)$ and we do not discuss this case further. Instead, we focus on the two-dimensional problem (corresponding to three-dimensional flow under the impactor), considering both the evolution of the contact set via the solution of the free boundary problem (1.2) - (1.4) and the dynamics of the splash sheet ejected from the turnover curve. As shown in [11], to leading order in $\epsilon, \delta$, the thickness of the sheet ejected from $(x, y)$ on $t=\omega(x, y)$ is $\epsilon^{2} H(x, y, t)$ and its velocity is $\mathbf{V}(x, y, t) / \delta$, where

$$
H=\frac{1}{4}(f(x, y)-s(t))^{2}, \mathbf{V}=2 v_{n} \mathbf{n},
$$

and $\mathbf{n}$ is the outward unit normal to the turnover curve in the $(x, y)$ plane. Although it is not the main concern of this paper, we remark that the main open question concerning the splash sheet dynamics is whether it separates from the body and if so where, some extra physics being required. However, it is clear that the fluid particles follow ballistic paths (confined to the body) before and after separation; given the location of the separation point, $[\mathbf{8}, \mathbf{1 6}]$ describe several possible separation mechanisms.

In $\S 2$ we consider the relationship between our free boundary problem and the wellknown squeeze film problem for a viscous fluid, and we discuss the linear stability of our problem in this context. In $\S 3$, we discuss an inverse method which allows us to construct explicit solutions to $(1.2)$ - (1.4) for certain body profiles and to find criteria under which the splash sheet can 'split' so that it leaves 'dry' or 'unsplashed' regions outside the turnover curve, with applications to the design of tyre tread. In $\S 4$, we consider a variational inequality formulation of the problem, show that certain 'moment' integrals over $\Omega(t)$ are constant and derive the Schwarz function formulation. Finally, we conclude in $\S 5$ with a discussion of directions for future work.

\section{Relationship with viscous squeeze film flows}

The two-dimensional version of the problem is closely related to squeeze film flows in a Hele-Shaw cell. We recall that in this model [15] a blob of viscous fluid trapped between two parallel plates separated by a distance $h(t)$ moves as the plates are squeezed together 
or pulled apart. For small separations, and sufficiently slow flow, the suitably scaled pressure $p(x, y, t)$ averaged across the gap satisfies

$$
\nabla^{2} p=\frac{\dot{h}}{h^{3}},
$$

in the fluid region $\Omega(t)$, which corresponds to our contact set, with

$$
p=0, \quad-h^{2} p_{n}=v_{n}
$$

on $\partial \Omega(t)$, corresponding to our turnover curve. The relationship between (1.2) - (1.4) and (2.1) - (2.2) is now clear: the functions of time differ but they can in fact be made identical by a suitable time change combined with a rescaling of $p$ (or $\phi$ ), leaving the essential new feature that for our problem the coefficient of $v_{n}$ in the kinematic condition (1.4) is spatially inhomogeneous. Later in the paper we shall use some techniques from the squeeze film problem in the context of the impact problem; for now, we just remark that the case $\dot{s}>0$, corresponding to fluid-entry, is analogous to $\dot{h}<0$, corresponding to an expanding squeeze film flow. As shown in [15], the latter is generally a well-posed problem, with a stable free boundary, whereas the case $\dot{h}>0$ is ill-posed, unstable and prone to finite-time blow up. This observation provides indirect support for the distinction between fluid-entry (stable, well-posed) and fluid exit (unstable, ill-posed) drawn in [1]. In more detail, a local in space and time linear stability analysis of perturbations proportional to $\exp (i k \nu+\sigma(k ; \mathbf{x}(t), t))$, where $k$ is the wavenumber and $\nu$ the tangential coordinate, gives the instantaneous growth rate

$$
\frac{\partial \sigma}{\partial t}(k ; \mathbf{x}(t), t)=-|k| v_{n}-\frac{\mathrm{d}}{\mathrm{d} t} \ln (f(\mathbf{x}(t))-s(t)) \quad \text { for } \quad \mathbf{x}(t) \in \Gamma(t)
$$

(the corresponding growth rate for $(2.1)-(2.2)$ is $-|k| v_{n}+\mathrm{d} / \mathrm{d} t(\ln h)$ for $\left.\mathbf{x}(t) \in \partial \Omega(t)\right)$. Thus, the flow is linearly stable if and only if the turnover curve is moving up the body; note that the large wavenumber limit of (2.3) is in agreement (in the sense that an advancing (retreating) turnover curve is stable (unstable)) with the linear stability analysis in $[\mathbf{1}]$ of the local travelling wave solution in a small neighbourhood of the jet root.

In the remainder of this paper we focus on the entry problem for which $\dot{s}>0$, so the trivial transformation $\phi=\dot{s} \hat{\phi}(x, y, \tau), \tau=s(t)$ implies that we may set $s(t)=t$ in $(1.2)$ - (1.4), without loss of generality.

\section{The inverse method}

We now present a method for calculating body shapes and turnover curves corresponding to a given solution of Poisson's equation (1.2). Suppose that $F(\cdot)$ is a twice continuously differentiable function of its argument. We first observe that

$$
\phi(x, y, t)=F(\omega(x, y))-F(t)
$$

automatically satisfies the boundary condition (1.3). Furthermore, if $F$ is invertible, so that

$$
\omega(x, y)=F^{-1}(G(x, y)) \quad \text { where } \quad G(x, y):=F(\omega(x, y))
$$


then by (1.2), $G$ satisfies Poisson's equation

$$
\nabla^{2} G=1 \quad \text { on } \quad \omega<t,
$$

and the initial condition, $\omega(0,0)=0$, provided

$$
F^{-1}(G(0,0))=0 \text {. }
$$

Since $v_{n}=|\nabla \omega|^{-1}$, the body profile is given by (1.4) and (3.2) as

$$
f(x, y)=\omega+F^{\prime}(\omega)|\nabla \omega|^{2}=F^{-1}(G)+\frac{|\nabla G|^{2}}{F^{\prime}\left(F^{-1}(G)\right)} .
$$

Hence, given a solution $G(x, y)$ of Poisson's equation (3.3), one may specify $F$ subject to (3.4), calculate $\omega$ from (3.2) and $f$ from (3.5), and finally check that the resulting solution is physically acceptable; we require $\omega<f$ for the turnover curve to lie above the undisturbed free surface, i.e. for the validity of the underlying asymptotics, and, for example, that both are smooth and monotonic increasing with radial distance from the origin.

We can also use this method to calculate the leading-order thickness and velocity of the splash root, for which (1.5) and (3.1) imply

$$
H=\frac{1}{4}|\nabla G|^{4}, \mathbf{V}=2\left|\frac{F^{\prime}\left(F^{-1}(G)\right)}{\nabla G}\right| \mathbf{n} .
$$

For our purposes the most important implication is that the splash sheet thickness vanishes at critical points of $G$ where $\nabla G=\mathbf{0}$, corresponding to non-smooth points of the turnover curve (which is also given by $G(x, y)=F(t)$ ). We investigate this phenomenon below, having presented a simple explicit example of the inverse method to demonstrate the procedure.

\subsection{Entry of an elliptic paraboloid}

As an example, we take

$$
G(x, y)=\frac{1}{2}\left(k x^{2}+(1-k) y^{2}\right),
$$

where $k \in[0,1]$. We let $F(t)=t / \alpha$ where $\alpha>0$, then by $(3.1)-(3.3)$,

$$
\phi(x, y, t)=\frac{1}{\alpha}(\omega(x, y)-t), \omega(x, y)=\left(\frac{x}{a_{1}}\right)^{2}+\left(\frac{y}{a_{2}}\right)^{2}, f(x, y)=\left(\frac{x}{A_{1}}\right)^{2}+\left(\frac{y}{A_{2}}\right)^{2},
$$

where $A_{j}$ and $a_{j}$ are given by

$$
\frac{1}{a_{1}^{2}}=\frac{\alpha k}{2}, \frac{1}{a_{2}^{2}}=\frac{\alpha(1-k)}{2}, \frac{1}{A_{1}^{2}}=\alpha\left(\frac{k}{2}+k^{2}\right), \frac{1}{A_{2}^{2}}=\alpha\left(\frac{(1-k)}{2}+(1-k)^{2}\right) .
$$

Hence, as in the infinite depth case [14], an entering elliptic paraboloid has a similarity solution in which distances scale with the square root of time and the turnover curve is an ellipse. The constant $\alpha\left(A_{1}, A_{2}\right)$ allows the impactor to be any elliptic paraboloid and is the unique positive root of the quartic in $\alpha$ obtained by eliminating $k$ from the last two equations in (3.9). If a cross-section of the entering elliptic paraboloid has eccentricity 


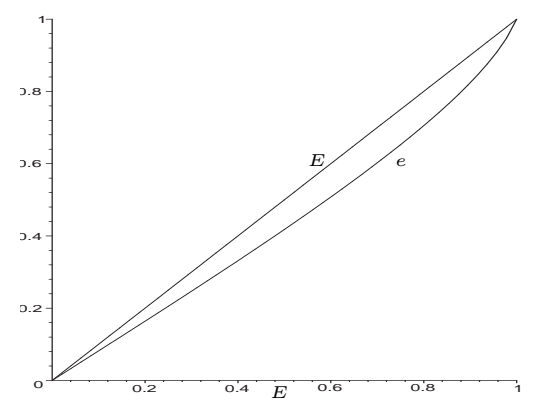

Figure 2. The eccentricity $e$ of the turnover curve as a function of the eccentricity $E$ of a cross-section of the entering elliptic paraboloid.

$E=\left(1-A_{2}^{2} / A_{1}^{2}\right)^{1 / 2}\left(A_{1}>A_{2}\right)$ and the turnover curve has eccentricity $e=\left(1-a_{2}^{2} / a_{1}^{2}\right)^{1 / 2}$ $\left(a_{1}>a_{2}\right)$, then eliminating $\alpha$ and $k$ from (3.9) implies

$$
e^{2}=1+\frac{E^{2}}{6}-\sqrt{1-E^{2}+\frac{E^{4}}{36}},
$$

which we plot in figure 2. As in the infinite depth case [14], $e<E$ for $0<E<1$, so the elliptic turnover curve is less elongated than a cross-section of the impacting elliptic paraboloid.

The splash root thickness and velocity are given by (3.6) as

$$
H=\frac{4 t^{2}}{\alpha^{4}}, \mathbf{V}=\frac{k x^{2}+(1-k) y^{2}}{k^{2} x^{2}+(1-k)^{2} y^{2}} \mathbf{n},
$$

in contrast to the infinite depth case in which, to lowest order, the jet thickness is not uniform in $x$ and $y$.

\subsection{Splitting the splash sheet}

We now consider the properties of the splash sheet within the framework of the inverse method. From (3.3),

$$
\nabla G \text { is harmonic, }
$$

and, since $\nabla G$ is not identically zero by (3.3), the spray sheet thickness (3.6a) cannot vanish on any subset of the turnover curve $\omega(x, y)=t$ that contains a limit point, i.e. if the thickness does vanish, it must do so at an isolated point. On physical grounds we also anticipate that for acceptable solutions

$$
F^{\prime}(t)>0
$$

$\omega$ and its first partial derivatives are bounded.

Since $\nabla G=F^{\prime}(\omega) \nabla \omega$, conditions $(3.11)-(3.13)$ imply that the splash root thickness (3.6a) vanishes at the isolated point $\left(x^{*}, y^{*}\right)$ if and only if $\nabla \omega\left(x^{*}, y^{*}\right)=\mathbf{0}$. Moreover, since $v_{n}=|\nabla \omega|^{-1}$, the normal velocity of the turnover curve is unbounded at such points and therefore the thickness vanishes if and only if the turnover curve develops an isolated non-smooth point, possibly a corner or a cusp. By (3.5), the body hits the free surface 

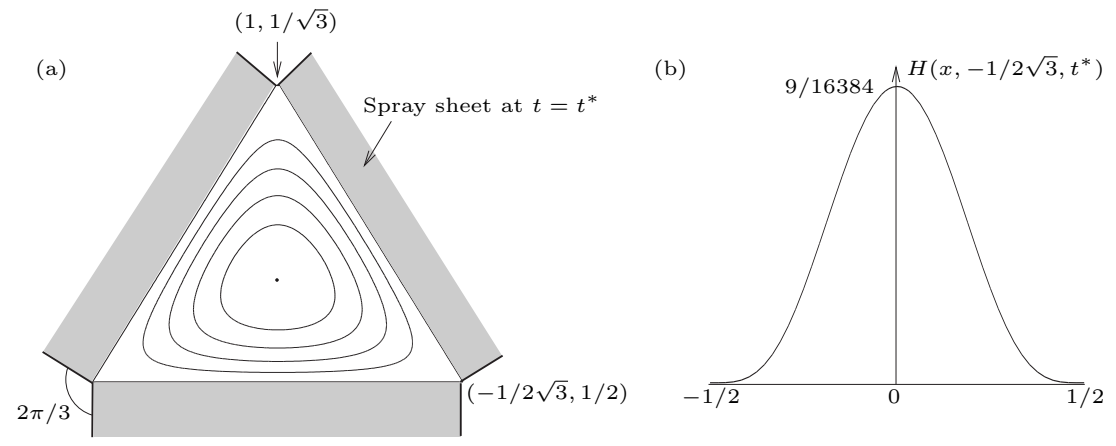

Figure 3. (a) Contour plot of the turnover curve $\omega(x, y)=t$ for $t=$ $0, t^{*} / 5,2 t^{*} / 5,3 t^{*} / 5,4 t^{*} / 5, t^{*}$. (b) Plot of the spray sheet thickness $H$ on the horizontal edge of the equilateral triangle $\omega(x, y)=t^{*}$. Solid lines indicate edges along which the splash sheet splits.

and is horizontal at such critical points, i.e. $f\left(x^{*}, y^{*}\right)=\omega\left(x^{*}, y^{*}\right)$ and $\nabla f\left(x^{*}, y^{*}\right)=\mathbf{0}$, which is in accordance with physical intuition.

In constructing such a turnover curve, $\omega(x, y)=t$, we note that it may also be written $G(x, y)=F(t)$ by (3.2). Hence, the inverse method allows us to construct the body profile that results in a given (non-smooth) turnover curve, $\Gamma^{*}$ say, provided we can solve (3.3) with the additional boundary condition

$$
G(x, y)=F\left(t^{*}\right) \quad \text { for } \quad(x, y) \in \Gamma^{*} .
$$

In this case the splash root thickness vanishes and therefore the sheet splits at non-smooth points on $\Gamma^{*}$ at time $t=t^{*}$, where $t^{*}$ is determined by (3.4).

For example, suppose we specify $\Gamma^{*}$ to be a simple closed polygon, then it is well known that (3.3) and (3.14) have a polynomial solution if and only if $\Gamma^{*}$ is an equilateral triangle (see, for example, [10] page 19). In this case, if $\partial \Omega$ is the equilateral triangle with unit side bounded by $y=-1 / 2 \sqrt{3}$ and $y \pm \sqrt{3} x=1 / \sqrt{3}$ (so there is symmetry in the $y$-axis and the centroid is at the origin), then

$$
G(x, y)=F\left(t^{*}\right)-\frac{1}{2 \sqrt{3}}(y+1 / 2 \sqrt{3})(y+\sqrt{3} x-1 / \sqrt{3})(y-\sqrt{3} x-1 / \sqrt{3}) .
$$

If we take $F(t)=t$, then $\omega=G, G(0,0)=0$ provided $t^{*}=\frac{1}{36}$ and by (3.5) the body is the quartic

$$
f(x, y)=\frac{1}{2}\left(x^{2}+y^{2}\right)+\frac{2}{\sqrt{3}} y\left(3 x^{2}-y^{2}\right)+\frac{3}{2} x^{2} y^{2}+\frac{3}{4}\left(x^{4}+y^{4}\right) .
$$

In figure 3 , we plot the contours $\omega(x, y)=t$ for six equally spaced times from $t=0$ to the critical time $t=t^{*}$ and the spray sheet thickness on the horizontal edge of $\omega(x, y)=t^{*}$, namely

$$
H\left(x,-1 / 2 \sqrt{3}, t^{*}\right)=\frac{9}{16384}\left(1-4 x^{2}\right)^{4} \quad \text { for } \quad|x|<1 / 2 .
$$

The velocity of the fluid ejected into the spray sheet is normal to the turnover curve, so when the sheet splits at a corner, one might expect the edges of the split sheet will span an angle of $2 \pi / 3$, as depicted in figure 3 . We plot $f(x, y)$ for $\omega(x, y)<t^{*}$ in figure 4 . 


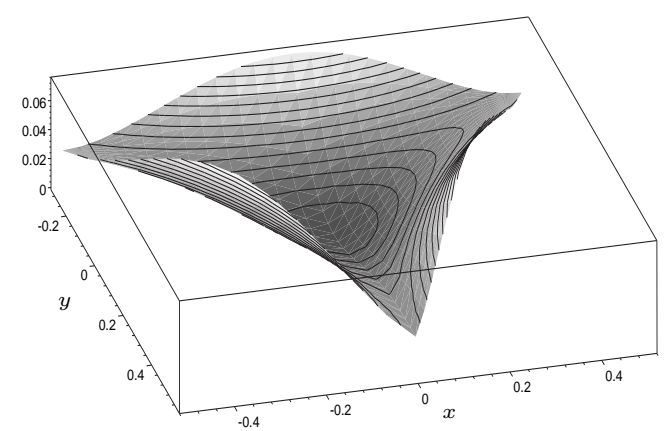

Figure 4 . Plot of the body profile $z=f(x, y)$ on $\omega(x, y)<t^{*}$.

Although the body profile is not convex, it is monotonic increasing with radial distance from the origin and is therefore physically acceptable.

Finally, we remark that the inverse method may be a useful design tool because, within the constraints discussed above, it allows us to specify the location of the turnover curve and in particular at which points the splash sheet will split. For example the $1 \mathrm{~mm}$ scale structure of tyre tread is designed to eject water into grooves that run around the tyre, thereby increasing contact with the road and therefore reducing the likelihood of hydroplaning. The analysis above suggests the non-smooth points of $G$ should be aligned with the grooves thereby forcing the impacting fluid into them. Of course, the simplifications we have made mean that we can expect our model to be more indicative of the fluid behaviour in such a situation than a precise quantitative tool.

\section{Direct approaches}

We now turn to a direct approach to the free boundary problem (1.2) - (1.4). We begin by making the observation that a variation inequality formulation is possible, although the resulting problem is not standard. We then discuss an analogue of Richardson's moments for the Hele-Shaw problem, and consider a formulation involving the Schwarz function for the free boundary.

\subsection{Variational inequality formulation}

The velocity potential $\phi(x, y, t)$ is defined on $\omega(x, y)<t$, so we define the displacement potential by

$$
\Upsilon(x, y, t)=-\int_{\omega(x, y)}^{t} \phi(x, y, \tau) \mathrm{d} \tau .
$$

A simple calculation using (1.3) shows that for $\omega<t$,

$$
\nabla^{2} \Upsilon=-\int_{\omega}^{t} \nabla^{2} \phi \mathrm{d} \tau+[\nabla \omega \cdot \nabla \phi]_{\omega=t}
$$

Since $\phi$ satisfies Poisson's equation (1.2) and on $\omega=t$,

$$
\nabla \omega \cdot \nabla \phi=|\nabla \omega| \mathbf{n} \cdot \nabla \phi=f-\omega,
$$


by (1.4), we deduce

$$
\nabla^{2} \Upsilon=\omega-t+f-\omega=f-t \text { for } \omega<t .
$$

Also, by (1.3) and (1.4),

$$
\Upsilon=\frac{\partial \Upsilon}{\partial n}=0 \quad \text { on } \quad \omega=t .
$$

On physical grounds, we assume the pressure is non-negative on the interior region, i.e.

which implies that, since $\phi=\Upsilon=0$ at $t=0$,

$$
-\frac{\partial \phi}{\partial t} \geq 0 \text { for } \omega<t
$$

$$
\Upsilon \geq 0 \quad \text { on } \omega<t .
$$

Morover, assuming that the waterline $f(x, y)=t$ lies inside the turnover curve $\omega(x, y)=$ t, i.e. $f>\omega$, and that $f$ is monotonic increasing with radial distance from the origin implies

$$
f-t \geq 0 \text { on } \omega>t .
$$

Finally, extending $\Upsilon$ to be zero on the exterior region $\omega>t$, i.e.

$$
\Upsilon=0 \text { on } \omega>t,
$$

one may formulate the free boundary problem (4.2) - (4.3) together with the conditions (4.4) - (4.6) as a variational inequality, namely

$$
\iint_{\Re^{2}} \nabla \Upsilon \cdot \nabla(v-\Upsilon) \mathrm{d} x \mathrm{~d} y \geq \iint_{\Re^{2}}(v-\Upsilon)(t-f) \mathrm{d} x \mathrm{~d} y
$$

for all non-negative square integrable $v$. However, since $f(x, y)-t<0$ inside the waterline $f(x, y)=t$, it is not amenable to classical theory.

\subsection{Generalized Richardson moments}

It is well-known [12] that for the Hele-Shaw problem $(2.1)-(2.2)$, with $\dot{h}=0$ and prescibed singularities to drive the flow,

$$
\iint_{\Omega(t)} \psi(x, y) \mathrm{d} x \mathrm{~d} y
$$

evolves in a predictable way whenever $\psi$ is harmonic and this enables the domain to be recovered (for example, from the 'moments' for which $\left.\psi_{n}=(x+i y)^{n}, n=0,1, \ldots\right)$. In a similar vein, for harmonic $\psi(x, y)$, we have

$$
\begin{aligned}
\iint_{\Omega(t)}(f(x, y)-t) \psi(x, y) \mathrm{d} x \mathrm{~d} y & =\iint_{\Omega(t)} \nabla \cdot(\psi \nabla \Upsilon-\Upsilon \nabla \psi) \mathrm{d} x \mathrm{~d} y \\
& =\int_{\Gamma(t)} \psi \Upsilon_{n}-\Upsilon \psi_{n} \mathrm{~d} \nu \\
& =0
\end{aligned}
$$

where we recall $\nu$ is the tangential coordinate along $\omega=t$ and we have used (4.2) and the fact that $\psi$ is harmonic in the first line, Green's theorem in the second and (4.3) in 
the third. Letting $\psi=1$ implies conservation of mass, i.e. the displaced fluid lies in the contact set to lowest order, and letting $\psi=x+i y$ implies that the centre of mass of the contact set remains at the origin. It is not, as far as we are aware, known whether the turnover curve is uniquely determined by the modified Richardson moments

$$
\iint_{\omega<t}(f(x, y)-t)(x+i y)^{n} \mathrm{~d} x \mathrm{~d} y=0 \quad \text { for } \quad n=0,1,2, \ldots,
$$

except in the simplest of self-similar cases, although with suitable qualification on the continuity of their time evolution it appears likely. For example, if $f$ and $\omega$ are given by (3.8), then taking $n=0$ and $n=2$ in (4.8) implies $a_{j}<A_{j}$ are determined in terms of $A_{j}$ by the pair of algebraic equations

$$
\begin{aligned}
4 A_{1}^{2} A_{2}^{2} & =a_{1}^{2} A_{2}^{2}+A_{1}^{2} a_{2}^{2}, \\
A_{1}^{2} a_{1}^{2} a_{2}^{2}+3 A_{2}^{2} a_{1}^{4}+6 A_{1}^{2} A_{2}^{2} a_{2}^{2} & =A_{2}^{2} a_{1}^{2} a_{2}^{2}+3 A_{1}^{2} a_{2}^{4}+6 A_{1}^{2} A_{2}^{2} a_{1}^{2},
\end{aligned}
$$

whose solution is in agreement with (3.9).

\subsection{Formulation in terms of the Schwarz function of $\Gamma(t)$}

In terms of the complex variable $z=x+i y$ the governing equation (1.2) is

$$
4 \frac{\partial^{2} \phi}{\partial z \partial \bar{z}}=1 \quad \text { for } \quad z \in \Omega
$$

where $\Omega$ denotes the (open) contact set in the complex plane and bar denotes the complex conjugate of a complex variable. The general solution to (4.10) is

$$
\phi=\frac{z \bar{z}}{4}+\Re(a(z, t))
$$

where $a(z)$ is an arbitrary analytic function on $\Omega$.

If the outward unit normal $\mathbf{n}=(\cos \theta, \sin \theta)$, then the boundary conditions (1.4) imply

$$
\begin{aligned}
0 & =-\sin \theta \frac{\partial \phi}{\partial x}+\cos \theta \frac{\partial \phi}{\partial y}, \\
v_{n}(f(x, y)-t) & =\cos \theta \frac{\partial \phi}{\partial x}+\sin \theta \frac{\partial \phi}{\partial y} .
\end{aligned}
$$

Subtracting $i$ times (4.12) from (4.13) gives the combined complex boundary condition

$$
2 \mathrm{e}^{i \theta} \frac{\partial \phi}{\partial z}=v_{n}(f(x, y)-t) \quad \text { on } \quad \Gamma .
$$

The Schwarz function of the free boundary $\Gamma$, which exists if and only if the boundary is analytic, is the unique function $g(z, t)$, analytic in some neighbourhood of $\Gamma$, such that

$$
\bar{z}=g(z, t)
$$

defines $\Gamma$. It is shown in $[\mathbf{3}]$ that

$$
\mathrm{e}^{-i \theta}=i \sqrt{g_{z}}, v_{n}=-\frac{i g_{t}}{2 \sqrt{g_{z}}} \quad \text { on } \quad \Gamma
$$

where subscripts denote partial derivatives. Hence, substituting (4.11) and (4.15) into 
(4.14) implies

$$
4 a_{z}+g=\left(f\left(\frac{1}{2}(z+g), \frac{1}{2 i}(z-g)\right)-t\right) g_{t} \quad \text { on } \quad \partial \Omega .
$$

Assuming $f$ is sufficiently regular, all terms in this equation are analytic in some neighbourhood of $\Gamma$, so by analytic continuation relation (4.16) holds wherever both sides are defined. Hence, the Schwarz function of an acceptable solution must satisfy the condition that

$$
\left(f\left(\frac{1}{2}(z+g), \frac{1}{2 i}(z-g)\right)-t\right) g_{t}-g \text { is analytic on } \Omega .
$$

Hence, to determine an exact solution one may postulate an analytic shape for the turnover curve $\partial \Omega$, write down its Schwarz function $g$ characterized by the parameters $\left\{c_{j}(t)\right\}$, say, then see if condition (4.17) uniquely determine $\left\{c_{j}(t)\right\}$.

Since $g_{z}$ cancels and does not appear in (4.17) one may integrate with respect to $t$ to obtain, applying the initial condition $g(z, 0)=0$,

$$
\operatorname{tg}(z, t)-\int_{0}^{g(z, t)} f\left(\frac{1}{2}(z+\eta), \frac{1}{2 i}(z-\eta)\right) \mathrm{d} \eta \quad \text { is analytic on } \Omega .
$$

\subsubsection{Example: The axisymmetric case}

Suppose the body has the axisymmetric profile $f(x, y)=f_{0}\left(\left(x^{2}+y^{2}\right)^{1 / 2}\right)$, where $f_{0}$ is monotonic increasing and $f_{0}(0)=0$. Furthemore, suppose the turnover curve $\Omega$ is a circle of radius $c(t)$ centred on the origin, so that the Schwarz function $g(z, t)=c(t)^{2} / z$. Applying (4.17), we find that

$$
\left(f_{0}\left(\left(\left(\frac{z+g}{2}\right)^{2}+\left(\frac{z-g}{2 i}\right)^{2}\right)^{1 / 2}\right)-t\right) g_{t}-g=\left(f_{0}(c)-t\right) \frac{2 c \dot{c}}{z}-\frac{c^{2}}{z}
$$

is analytic on $\Omega$ if and only if $c=\left(f\left(c_{0}\right)-t\right) 2 \dot{c}$. Integrating and applying the initial condition $c(0)=0$ implies

$$
\int_{0}^{c(t)}(f(r)-t) r \mathrm{~d} r=0
$$

which is the required law of motion. Further, $a_{z}=0$ and therefore

$$
\phi(x, y, t)=\frac{x^{2}+y^{2}-c(t)^{2}}{4}
$$

in order to satisfy (1.3).

\section{Conclusion}

We have described a novel free boundary problem for the contact set (corresponding to the high pressure region) of a blunt body impacting on shallow water. This problem has a mathematical structure which closely resembles that of the Hele-Shaw problem and the viscous squeeze film problem. We have exhibited several explicit solutions, and we have shown that weighted harmonic moments are conserved. We have also presented 
a nonlinear version of the Schwarz function treatment of the Hele-Shaw problem; it is possible that Cauchy transform methods, as discussed in $[\mathbf{2}, \mathbf{1 3}]$, may also prove fruitful in our case.

\section{Acknowledgements}

The authors are grateful to Prof. Bjorn Gustaffson, Dr. Peter Howell and the referees for helpful comments.

\section{References}

[1] Chapman, S. J., Gillow, K. A., Howison, S. D. \& Ockendon, J. R. 1997 Asymptotics of violent surface motion. Phil. Trans. Roy. Soc. Lon. A 355, 679-685.

[2] Crowdy, D. G. 2004 On a class of geometry-driven free boundary problems. SIAM J. App. Math. 62(3), 945-964.

[3] Davis, P. J. 1974 The Schwarz function and its applications. Carus Mathematical Monographs $\mathbf{1 7}$

[4] Fraenkel, L. E. \& McLeod, J. B. 1997 Some results for the entry of a blunt wedge into water. Phil. Trans. Roy. Soc. Lon. A 355, 523-535.

[5] Howison, S. D., Ockendon, J. R. \& Wilson, S. K. 1991 Incompressible water-entry problems at small deadrise angles. J. Fluid Mech. 222, 215-230.

[6] Howison, S. D., Morgan, J. D. \& Ockendon, J. R. 1997 A class of codimension-two free boundary problems. SIAM Review 39(2), 221-253.

[7] Howison, S. D., Ockendon, J. R. \& Oliver, J. M. 2002 Deep- and shallow-water slamming at small and zero deadrise angles. J. Eng. Math. 42, 373-388.

[8] Keller, J. B. \& GeER, J. 1973 Flows of thin streams with free boundaries. J. Fluid Mech. $\mathbf{5 9}, 417-432$.

[9] Korobkin, A. A. 1995 Impact of two bodies one of which is covered by a thin layer of liquid. J. Fluid Mech. 300, 43-58.

[10] Ockendon, H. \& Ockendon, J. R. 1995 Viscous Flow. Cambridge University Press.

[11] Oliver, J. M. 2002 Water-entry and related problems. D.Phil. Thesis, University of Oxford.

[12] Richardson, S. 1972 Hele-Shaw flows with a free boundary produced by the injection of fluid into a narrow channel. J. Fluid Mech. 56, 609-618.

[13] Richardson, S. 1981 Some Hele-Shaw flows with time-dependent free boundaries. J. Fluid Mech. 102, 263-278.

[14] Scolan Y. -M. \& Korobkin, A. A. Three-dimensional theory of water impact. Part 1. Inverse Wagner problem. J. Fluid Mech. 440, 293-326.

[15] Shelley, M. J., Tian, F. -R. \& Wlodarski, K. 1997 Hele-Shaw flow and pattern formation in a time-dependent gap. Nonlinearity 10, 1471-1495.

[16] Tayler, A. B. 1972 Singularities at flow separation points. Quart. J. Mech. Appl. Math. XXVI(2), 153-172.

[17] WagneR, H. 1932 Über stoß- und gleitvorgänge an der oberfläche von flüssigkeiten (Phenomena associated with impacts and sliding on liquid surfaces). Zeitschrift für Angewandte Mathematik und Mechanik 12, 193-215. 